Research Article

\title{
Error Compensation Technique for a Resistance-Type Differential Pressure Flow Sensor
}

\author{
Guimei Wang $(\mathbb{D}$, Tao Chu $(\mathbb{D}$, Lijie Yang $\mathbb{D}$, and Fang Sun \\ College of Mechanical and Equipment Engineering, Hebei University of Engineering, Handan 056038, China \\ Correspondence should be addressed to Lijie Yang; yanglijie255@163.com
}

Received 15 October 2017; Revised 5 January 2018; Accepted 23 January 2018; Published 29 April 2018

Academic Editor: Zongyao Sha

Copyright ( 2018 Guimei Wang et al. This is an open access article distributed under the Creative Commons Attribution License, which permits unrestricted use, distribution, and reproduction in any medium, provided the original work is properly cited.

\begin{abstract}
A flow sensor is designed based on resistance-type differential pressure flow (RDPF) method, and the flow data is measured during a coal gangue paste-filling process. The measurement error characteristics of a RDPF sensor are analyzed. Periodic and aperiodic errors are then modeled separately. The model for the periodic error is established by Fourier series approximation using least squares solution of an overdetermined equation to solve for the model parameters. The model for the aperiodic error is established using an online least squares support vector machine (LS-SVM) method. The cross-validation is used to solve model parameters. Simulations and experiments show that the dynamic measurement accuracy of the sensor is greatly improved by error compensation, thereby reducing filling material waste and improving the economic efficiency.
\end{abstract}

\section{Introduction}

Coal gangue paste filling is a backfill mining technique that has the dual aim of reducing environmental pollution caused by coal gangue and flyash and lessening the risk of subsidence [1]. As green filling mining technology, the paste-filling technique has the above advantages. However, with the long filling pipe, nonuniform velocity, filling paste of large particles (over $25 \mathrm{~mm}$ in diameter) impose significant uncertainities for the paste filling pipe flow detection. The efficiency of paste filling depends on the flow rate of the paste in the filling pipe; but up to now, it has been difficult to measure this rate with sufficient accuracy. The inaccuracy in the flow rate measurement has resulted in a great waste of filling material and excessive consumption of electricity and human resources, causing deleterious consequences for environmental issues and safety production.

At present, the sensors used for fluid flow measurement include differential pressure flowmeter, capacitive flowmeter, electromagnetic flowmeter, and ultrasonic flowmeter, and all of those are not suitable for measure multiphase fluid flow precisely [2]. Differential pressure flowmeter needs installation of an orifice plate or a shrink diameter pipe. The large diameter material has a serious side effect of aggregating damage on the orifice plate, which is likely to cause congestion accidents [3]. Capacitance flowmeter fails to capture measurement of fluid flow because the dielectric value of the paste is usually reflected by the dynamic changes of the concentration of the paste [2]. Electromagnetic flowmeter can be easily blocked, because the large diameter material accounted for about $50 \%$ of the filling material, resulting in distortion of the measurement results of the sensor $[2,3]$. Ultrasonic flowmeter sensor is unable to accurately detect flow information because only weak signals are received due to the high mass concentration of the filling paste and the serious viscous viscosity and scattering attenuation [4]. Because of these limitations in paste flow measurement, all the above flow sensors cannot be effectively used in detection for paste-filling flow.

A resistance-type differential pressure flow (RDPF) sensor has been developed to achieve precise measurements. Noncontact and real-time flow measurement in the paste- 


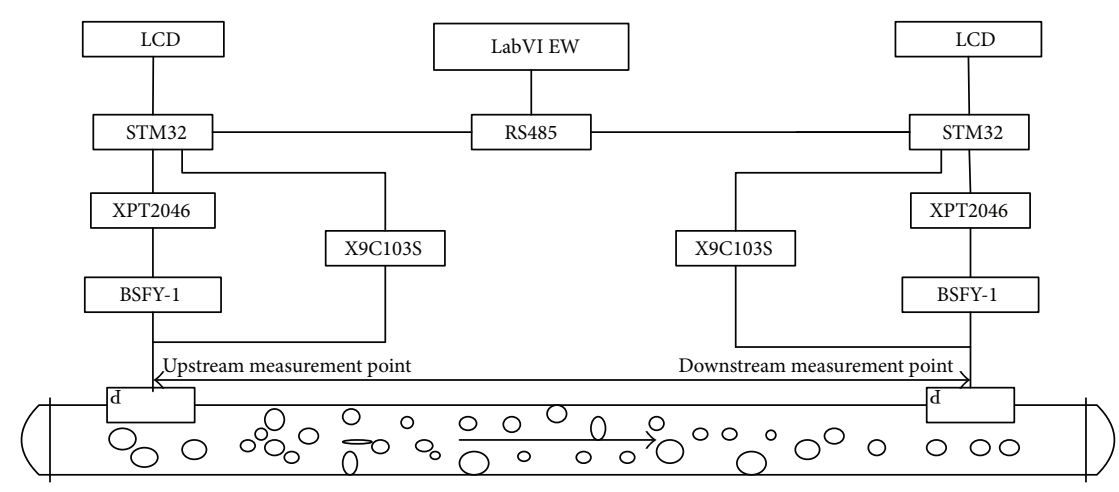

FIgURE 1: Schematic diagram of the RDPF sensor.

filling pipe is realized by the sensor. However, there is much space for improvement in the measurement error of the RDPF sensor. Thereby, error compensation model is built to compensate error. In recent years, both neural network theory and fuzzy theory have been applied to error modeling, but these two approaches are appropriate only for offline modeling and are not applicable to online error compensation [5-7]. Common methods that are suitable for online modeling include autoregressive modeling, minimum support vector machine modeling, and Bayesian dynamic model theory [8-13]. The paste flow in the pipeline changes periodically, which must be taken into account when building error compensation models to achieve sufficient accuracy [14-16]. Because the above models are not ideal for error compensation of RDPF sensor, a new compensation error method is proposed [17-19].

\section{RDPF Sensor and Analysis of Its Measurement Error Characteristics}

2.1. RDPF Sensor. Based on pressure difference detection principle, an indirect pipe flow measurement method is proposed by measuring the deformation of filling pipe. According to the pressure difference test theory of resistance strain gauge, the RDPF sensor is designed. The sensor includes a bridge measuring circuit, an amplifying circuit, an electric bridge automatic zeroing circuit, an analog/digital (A/D) conversion circuit, and a sample hold circuit. The analog signals from the strain gauge are converted into digital signals, and then the digital signals are uploaded through a communication module to a host computer which displays and stores the fill-pipe flow value with the software of LabVIEW. The principle of the RDPF sensor is shown in Figure 1.

2.2. Measurement Error Analysis for the RDPF Sensor. Mined-area filling capacity is regarded as the exact value of the paste flow, and it is compared with flow value measured by the RDPF sensor without the use of any error compensation. The result shows that the accuracy of the sensor is low, with a relative error of about $5 \%$ compared to the value of mined-area filling capacity.

The flow rate in the paste-filling pipe following pipe pressure undergoes periodic changes, which is shown in Figure 2. The errors in the sensor can be divided into three

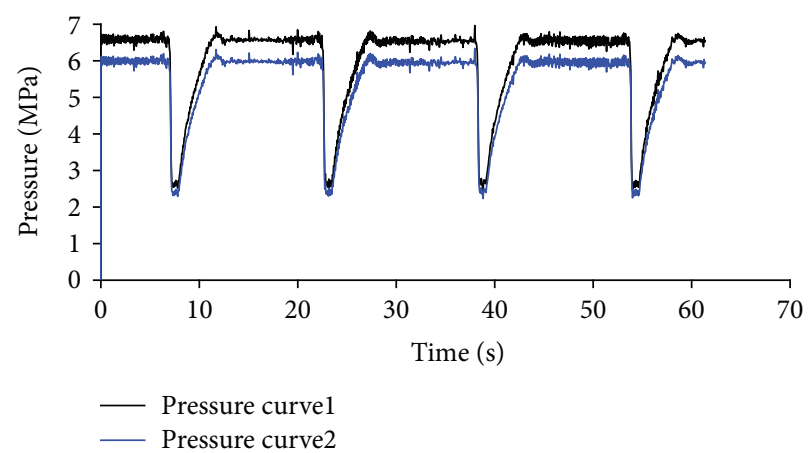

FIgURe 2: Measured value of pressure of paste-filling pipeline. Pressure curve1: pressure curve at upstream measuring point. Pressure curve2: pressure curve at downstream measurement point.

components: systematic error, random error, and gross error [20]. The gross error is mainly caused by accidental factors in the measurement process, such as strong interference, vibration, and human factors. The gross error can be eliminated by previous experience before building the compensation error model. The systematic error mainly consists of three components: pipe flow modeling error, strain gauge error due to the effects of the paste, and measurement error caused by pipe wear [21].

(1) The systematic error is produced because the rising stage of each cycle is approximated as a linear process for the pipe flow model.

(2) The systematic error is also caused by the difference of strain gauge-pasted angle and adhesive coating thickness of RDPF.

(3) When the paste velocity is low, there is serious pipe wear in straight sections of the pipe, and then the system error is also caused by the abrasion of the filling pipeline.

The flow sensor is installed on a straight pipe section with high paste-filling pressure. The installation position of the sensor is close to the paste-filling station. The random error is caused by the environmental noise and electromagnetic interference in this region. 


\section{Error Modeling}

By comparison of the paste-filling capacity measured by the flow sensor with the paste-filling capacity of the mined area, the measurement error of the sensor $\Delta \delta(t)$ is obtained. According to the real-time flow curve in Figure 2, the filling flow changed in a periodic fashion follows the filling stroke of the filling pump. The measurement error can then be expressed as

$$
\Delta \delta(t)=\Delta \delta_{u}(t)+\Delta \delta_{e}(t)
$$

where $t$ is the measurement time, $\Delta \delta_{u}(t)$ is the periodic error component, and $\Delta \delta_{e}(t)$ is the aperiodic error component.

3.1. Periodic Error Compensation Model. Through mathematical analysis of the systematic error, it is known that the periodic error in the flow sensor satisfies a Dirichlet condition (DC) in the period $[0, T]$, and therefore the periodic error in the dynamic measurements can be decomposed into an infinite series of linear combinations of trigonometric functions:

$$
\begin{aligned}
\Delta \delta_{u}(t)= & a_{0}+a_{1} \cos \omega_{0} t+b_{1} \sin \omega_{0} t+a_{2} \cos \omega_{0} t \\
& +b_{2} \sin \omega_{0} t+\cdots=a_{0}+\sum_{h=1}^{\infty}\left(a_{h} \cos \omega_{0} t+b_{h} \sin \omega_{0} t\right),
\end{aligned}
$$

where $\omega_{0}$ is fundamental wave angle frequency, and $a_{0}$ , $a_{1}, a_{2}, \ldots, a_{n}, b_{1}, b_{2}, \ldots, b_{n}$ are Fourier coefficients of signals.

Using trigonometric identities, formula (2) can be rewritten as follows:

$$
\Delta \delta_{u}(t)=A_{0}+\sum_{h=1}^{\infty} A_{h} \cos \left(h \omega_{0} t-\phi_{h}\right),
$$

where $A_{0}$ is the amplitude of the DC component, $A_{h}=$ $\sqrt{a_{h}^{2}+b_{h}^{2}}$ are the amplitudes of the frequency components, and $\phi_{h}=\arctan \left(a_{h} / b_{h}\right)$ are the phase angles of the frequency components. Therefore, the dynamic model of measurement error for the sensor is built after the amplitude and phase angle for each frequency are determined.

In the dynamic measurement of the paste flow, $k+1$ data points are measured in each period. The measurement period is discretized according to the equidistant measurement method to obtain $\Delta \delta_{u}(t)$, and the time value of the measurement point can be expressed as $t_{i}=i t /(k+1), i=0$, $1,2, \ldots, k$.

Formula (3) can be expressed in matrix form as follows:
TABle 1: Amplitude and phase angle of each harmonic.

\begin{tabular}{lcc}
\hline Number of harmonics $n$ & $\begin{array}{c}\text { Harmonic } \\
\text { amplitude } A_{n}\end{array}$ & $\begin{array}{c}\text { Harmonic } \\
\text { angle } \varphi_{n}\end{array}$ \\
\hline 0 & -181.442 & \\
1 & 5.767899 & 6.362538 \\
2 & 215.75 & 1.458583017 \\
3 & -495.472 & 2.039259482 \\
4 & 325.7386 & 1.367623042 \\
5 & 155.6761 & 3.624029617 \\
\hline
\end{tabular}

Theoretically, in the formula (2), an infinite number of frequency components are superimposed, but when the relative error between the values measured by the flow sensor and mined-area filling capacity is less than $1 \%$, the measurement accuracy is completely satisfactory, and the summation over frequency components can be truncated.

3.2. Aperiodic Error Dynamic Compensation Model. Based on the dynamic data exchange (DDE) technique and the least squares support vector machine (LS-SVM) method, a dynamic compensation model of aperiodic error is established [22]. The LS-SVM method employs a support vector machine to convert inequality constraints into equality constraints. A nonlinear mapping between the filling time and the aperiodic error in the filling flow is established by the method, and nonlinear laws hidden in the sample data is revealed. For nonlinear sample data $\left(x_{1}, y_{1}\right)$, $\left(x_{2}, y_{2}\right), \ldots,\left(x_{l}, y_{l}\right)$, using LS-SVM regression, the regression problem for the aperiodic error can be described as follows: 
TABLE 2: Sensor measured values.

\begin{tabular}{lcccc}
\hline Time/h & $\begin{array}{c}\text { Mined-area filling } \\
\text { capacity } / \mathrm{m}^{3}\end{array}$ & $\begin{array}{c}\text { Sensor measured value } \\
\text { (uncompensated) } / \mathrm{m}^{3}\end{array}$ & $\begin{array}{c}\text { Sensor measurement } \\
\text { (compensated for aperiodic error) } / \mathrm{m}^{3}\end{array}$ & $\begin{array}{c}\text { Sensor measurement (compensated for } \\
\text { periodic and nonperiodic errors) } / \mathrm{m}^{3}\end{array}$ \\
\hline 0.5 & 0.625 & 0.655 & 0.6298 & 0.6251 \\
1 & 1.24 & 1.3 & 1.2475 & 1.2402 \\
1.5 & 1.845 & 1.935 & 1.8545 & 1.8452 \\
2 & 2.46 & 2.57 & 2.461 & 2.46 \\
2.5 & 3.06 & 3.195 & 3.0568 & 3.0599 \\
3 & 3.66 & 3.84 & 3.6721 & 3.6603 \\
3.5 & 4.26 & 4.465 & 4.2668 & 4.2602 \\
4 & 4.87 & 5.105 & 4.8677 & 4.8699 \\
4.5 & 5.485 & 5.745 & 5.4845 & 5.485 \\
5 & 6.09 & 6.375 & 6.0825 & 6.0898 \\
5.5 & 6.69 & 7 & 6.675 & 6.6897 \\
6 & 7.3 & 7.645 & 7.2869 & 7.2997 \\
\hline
\end{tabular}

$$
\begin{array}{r}
\min J(\boldsymbol{\omega}, \xi)=\frac{1}{2} \boldsymbol{\omega}^{T} \boldsymbol{\omega}+\gamma \frac{1}{2} \sum_{i=1}^{l} \xi_{i}^{2}, \\
\text { s.t. } \quad y_{i}=\boldsymbol{\omega}^{T} \varphi\left(x_{i}\right)+b+\xi_{i}, \\
i=1, \ldots, l,
\end{array}
$$

where $x_{1}, x_{2}, \ldots, x_{i}$ are the input variables, $y_{1}, y_{2}, \ldots, y_{i}$ are the output expected values, $\xi_{1}, \xi_{2}, \ldots, \xi_{i} \in R$ are the error variables, $\varphi(x): R^{n} \rightarrow R^{n h}$ is the kernel space mapping function, $\omega \in R^{n h}$ is a right vector, $\gamma$ is an adjustable parameter, and $b$ is the amount of deviation. Then, the Lagrangian function is introduced.

$$
L=\frac{1}{2} \omega^{T} \omega+\gamma \frac{1}{2} \sum_{i=1}^{l} \xi_{i}^{2}-\sum_{i=1}^{l} a_{i}\left[\omega^{T} \varphi\left(x_{i}\right)+b+\xi_{i}-y_{i}\right],
$$

where $a_{i}(i=1,2, \ldots, l)$ is Lagrange multiplies. According to the necessary conditions for the existence of extreme values, $\partial L / \partial \omega=0, \partial L / \partial b=0, \partial L / \partial \xi_{i}=0$, and $\partial L / \partial a=0$, the following formula is gotten:

$$
\begin{aligned}
& \omega=\sum_{i=1}^{l} a_{i} \varphi\left(x_{i}\right), \\
& \sum_{i=1}^{l} a_{i}=0, \\
& a_{i}=\gamma \xi_{i}, \\
& y_{i}=\omega^{T} \varphi\left(x_{i}\right)+b+\xi_{i}, \\
& i=1,2, \ldots, l .
\end{aligned}
$$

The following formula is obtained by eliminating $\omega$ and $\xi$ of formula (7),

$$
\beta_{l} A_{l}=Y_{l}
$$

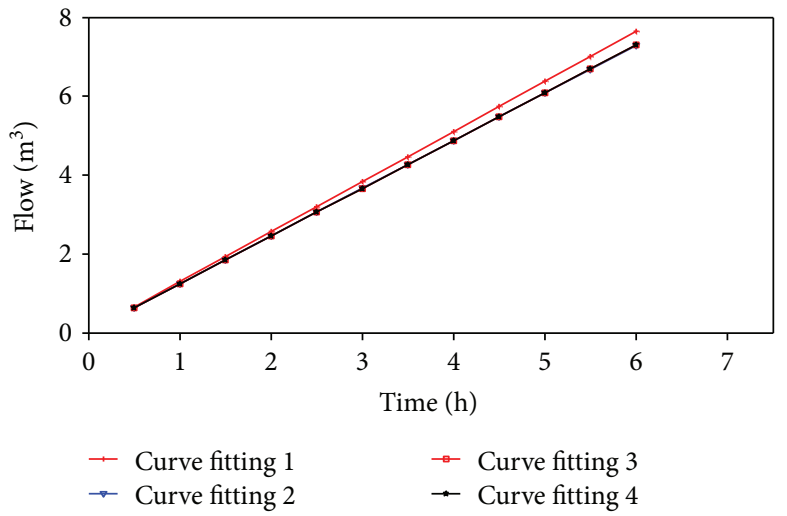

FIgURe 3: Flow values before and after error compensation. Curve fitting 1: sensor measured value (uncompensated). Curve fitting 2: sensor measurement (compensated for aperiodic error). Curve fitting 3: sensor measurement (compensated for periodic and nonperiodic errors). Curve fitting 4: mined-area filling capacity.

$$
\begin{aligned}
\beta_{l} & =\left[\begin{array}{cccc}
0 & 1 & \ldots & 1 \\
1 & K\left(x_{1}, x_{1}\right)+\frac{1}{\gamma} & \ldots & K\left(x_{1}, x_{l}\right) \\
\vdots & \vdots & \vdots & \vdots \\
1 & K\left(x_{l}, x_{1}\right) & \ldots & K\left(x_{l}, x_{l}\right)+\frac{1}{\gamma}
\end{array}\right], \\
A_{l} & =\left[\begin{array}{c}
b \\
a_{1} \\
\vdots \\
a_{l}
\end{array}\right], \\
Y_{l} & =\left[\begin{array}{c}
0 \\
y_{1} \\
\vdots \\
y_{l}
\end{array}\right]
\end{aligned}
$$

where $K\left(x, x_{i}\right)$ is kernel function. 


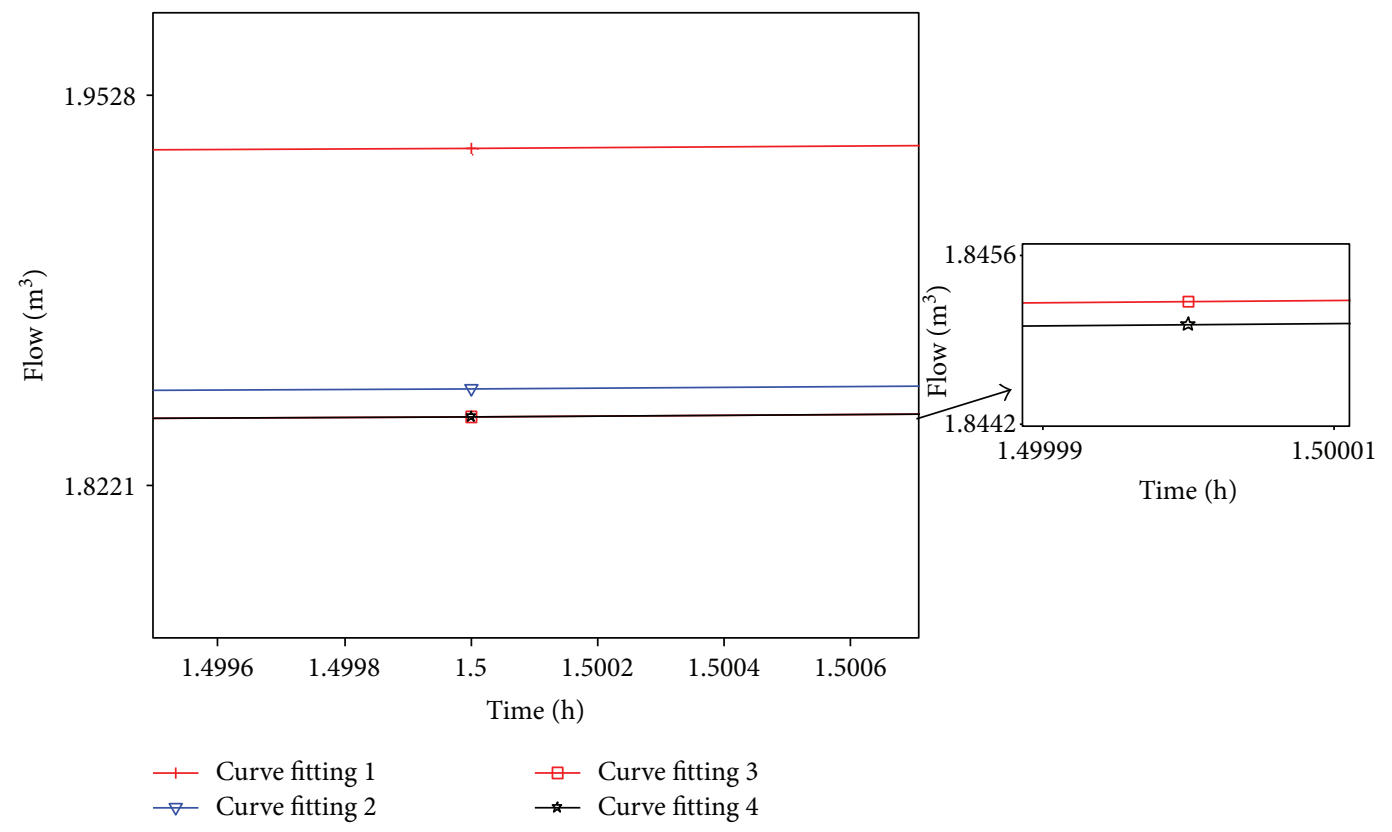

FIGURE 4: Local enlargement of Figure 3.

Linear kernel functions, polynomial kernel functions, and the radial basis function (RBF) kernel functions are commonly used kernel functions. The kernel function must satisfy Mercer's condition. Then, LS-SVM regression estimate using kernel function $K\left(x, x_{i}\right)$ can be expressed by formula (10). The values of $a_{i}$ - and $b$ are obtained by the least square method [23-25].

$$
f(x)=\sum_{i=1}^{l} a_{i} K\left(x, x_{i}\right)+b .
$$

\section{Results}

4.1. Determination of Model Parameters. The error of the Fourier series approximation model satisfies the filling requirement when it is less than $1 \%$. The error in the Fourier series approximation model meets the sensor measurement requirements when the fifth harmonic overlay is fitted by MATLAB. Using formula (7), the least squares principle is applied to solve for the amplitude and phase angle of each harmonic. The results are shown in Table 1.

The radial basis function $(\mathrm{RBF}) \quad K\left(x_{i}, x_{j}\right)=\exp [-$ $\left.\left(x_{i}-x_{j}\right)^{2} /\left(2 \sigma^{2}\right)\right]$ is selected as the kernel function. The cross-validation method is chosen for determining the parameter $\sigma$ in the RBF and the parameter $\gamma$ in the LS-SVM model. To decrease the search time of the two parameters, the kernel function $\sigma=\{20,10,5,2,1,0.1,0.01\}$ and the punishment constant $\gamma=\{200,100,50,20,10,5,2,1\}$ are chosen, thereby, the optimal parameter pairs $(\gamma=200, \sigma=0.1)$ are obtained by cross-validation. Then, acquisition time as the input, and the flow value measured by the sensor as the output, an aperiodic error compensation model is established. The square sum of the relative error is obtained, which is 0.0262 .

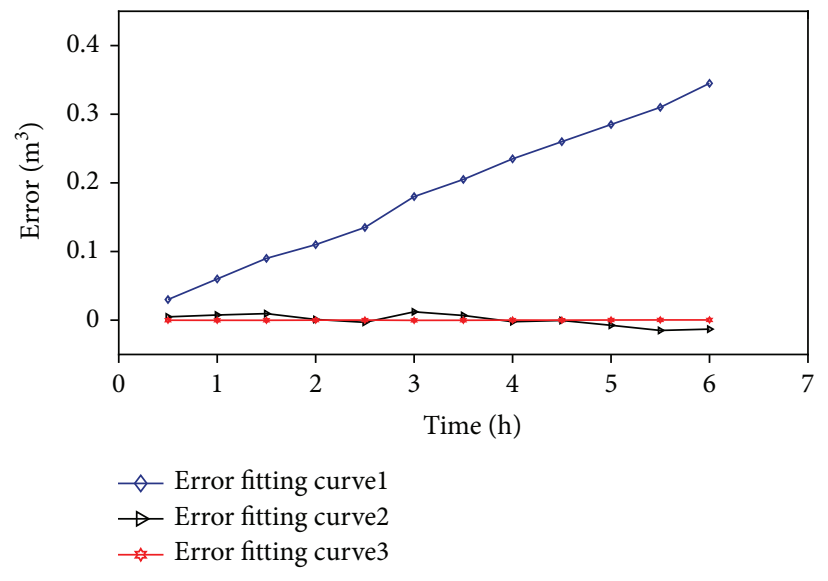

FIgURE 5: Error before and after error compensation. Error fitting curve1: measured error of sensor without compensated. Error fitting curve2: measured error of sensor with compensated for aperiodic. Error fitting curve3: measured error with compensated for periodic and nonperiodic errors.

4.2. Experimental Analysis. In coal gangue paste-filling process, the experimental platform of the sensor measurement accuracy is built. The sensor without compensation, the sensor of compensation for periodic error, and the sensor of compensation periodic error and aperiodic error are installed in the experimental pipeline. The measured flow values of three sensors are listed in Table 2.

The curve of flow values before and after error compensation is shown in Figure 3 after following the measured data in Table 2. Local enlargement of Figure 3 is shown in Figure 4. Measurement values with different compensation module are compared with mined-area filling capacity value (Figure 5). Seen form Figure 5, the measurement error increases with time without error compensation. When the Fourier series approximation method is used to compensate 
for the periodic error, the measurement error of the sensor is obviously reduced. When the LS-SVM method is used to compensate periodic error, the measurement error of the sensor is reduced greatly, which is changed little with time.

\section{Discussion}

Under different conditions, the measured curves and error curves of the sensor are shown in Figures 3-5. Seen from Figures 3 to 5 , the sensor has a large measurement error in the absence of compensation, but, when using the Fourier series approximation model to compensate for the periodic error, the error is greatly reduced, which shows that periodic error is the main component of the sensor measurement error. After compensation for both periodic and aperiodic error, the measurement error of the sensor fluctuates around zero, which indicates that the dynamic measurement accuracy of the sensor is greatly improved.

To guarantee dynamic compensation of aperiodic error, dynamic data exchange (DDE) can be used to realize realtime data exchange between MATLAB and the host computer. The flow value measured by the sensor and the mined-area filling capacity obtained through a data acquisition card are uploaded to the host computer. Models of the periodic and aperiodic errors are established in MATLAB using the above values, and the model parameters are calculated and then sent to the error compensation model to achieve dynamic compensation of measurement errors.

The value of RDPF sensor is related to the filling pipe pressure and the length and cross-sectional area of the filling pipe. When the discontent tube filling fault occurs, the crosssectional area of the filling paste in the tube is smaller than the pipe cross-sectional area, making the measured result deviating from the actual filling flow. Therefore, the filling failure of the discontent tube should be avoided to improve the measurement accuracy of the RDPF sensor.

\section{Conclusions}

The measurement errors of the RDPF sensor have been analyzed. The periodic error model is established by a Fourier series approximation method. The parameters of the periodic error model are obtained by solving an overdetermined matrix by the least squares method. The aperiodic error model is constructed using an online LS-SVM method, and the model parameters are solved by cross-validation. Experiments show that the method proposed in the paper can greatly reduce the measurement error of the sensor and improve its dynamic measurement accuracy.

\section{Conflicts of Interest}

The authors declare no conflict of interest.

\section{Authors' Contributions}

Lijie Yang and Guimei Wang conceived and designed the experiments. Tao Chu performed the experiments. Lijie Yang analyzed the data. Fang Sun contributed analysis tools. Tao Chu and Lijie Yang wrote and revised the paper.

\section{Acknowledgments}

This project was supported by the Hebei Province Science and Technology Support Program (16394102D) and the science and technology research project of Hebei Higher Education Institutions (QN2014171).

\section{References}

[1] J. G. Liu, "Study and practice of low-carbon ecological mining," Journal of China Coal Society, vol. 36, no. 2, pp. 317321, 2011.

[2] G. M. Wang and S. Zhang, "Paste filling pipe on-line detection system," Machine Tool \& Hydraulics, vol. 43, no. 8, pp. 161163, 2015.

[3] Y. J. Chen and B. W. Zhao, Integrated Mechanized Paste Filling Coal Mining Technology, Coal Industry Press, Beijing, China, 2012.

[4] D. Y. Wang, Analysis of Attenuation Characteristics of Ultrasonic Propagation in Mud, Shenyang University of Technology, 2014.

[5] S. Z. Sun and D. L. Peng, "Research on modeling and compensation technology for time-grating sensor's dynamic measurement errors," Journal of Mechanical Engineering, vol. 50, no. 22, pp. 10-15, 2014.

[6] S. Z. Sun and D. L. Peng, "Compensation for dynamic measurement errors of time grating sensor," Optics and Precision Engineering, vol. 23, no. 4, pp. 1114-1121, 2015.

[7] S. G. Gumarov and O. N. Korsun, "A method of determining the dynamic error of optical trajectory measurement stations," Measurement Techniques, vol. 54, no. 3, pp. 281-286, 2011.

[8] J. Guajardo, R. Weber, and S. F. Crone, "A study on the ability of support vector regression and neural networks to forecast basic time series patterns," International Federation for Information Processing Digital Library, vol. 217, no. 1, pp. 149-157, 2010.

[9] T. Zhang and W. H. Ye, “Temperature variable optimization for precision machine tool thermal error compensation on optimal threshold," Chinese Journal of Mechanical Engineering, vol. 26, no. 1, pp. 158-165, 2013.

[10] S. Q. Liu and R. Zhu, "System error compensation methodology based on a neural network for a micromachined inertial measurement unit," Sensors, vol. 16, no. 2, p. 175, 2016.

[11] F. J. Wang and Z. Y. Jia, “Time-varying position error compensation of machine tools based on dynamic fuzzy neural networks," Journal of Mechanical Engineering, vol. 47, no. 13, pp. 175-179, 2011.

[12] Y. Zhang and J. G. Yang, "Modeling for machine tool thermal error based on grey model preprocessing neural network," Journal of Mechanical Engineering, vol. 47, no. 7, pp. 134138, 2011.

[13] D. H. Wu, "Compensation for nonlinear dynamic system of transducer based on least squares support vector machine," Chinese Journal of Scientific Instrument, vol. 28, no. 6, pp. 1018-1023, 2007.

[14] H. Yang, K. Huang, I. King, and M. R. Lyu, "Localized support vector regression for time series prediction," Neurocomputing, vol. 72, no. 10-12, pp. 2659-2669, 2009. 
[15] Z. Jia, "Characteristics prediction method of electro-hydraulic servo valve based on rough set and adaptive neuro-fuzzy inference system," Chinese Journal of Mechanical Engineering, vol. 23, no. 2, p. 200, 2010.

[16] H. Yang, L. Zhang, J. Zhou, Y. Fei, and D. Peng, "Modelling of dynamic measurement error for parasitic time grating sensor based on Bayesian principle," Optics and Precision Engineering, vol. 24, no. 10, pp. 2523-2531, 2016.

[17] W. Lin, J. Z. Fu, Z. C. Chen, and Y. Z. Xu, "Modeling of NC machine tool thermal error based on adaptive best-fitting WLS-SVM," Journal of Mechanical Engineering, vol. 45, no. 3, pp. 178-182, 2009.

[18] J. Li, G. Hu, Y. Zhou, and J. A. SM, “A temperature compensation method for piezo-resistive pressure sensor utilizing chaotic ions motion algorithm optimized hybrid kernel LSSVM," Sensors, vol. 16, no. 10, p. 1707, 2016.

[19] J. Li, G. Hu, Y. Zhou, C. Zou, W. Peng, and J. A. Sm, "Study on temperature and synthetic compensation of piezo-resistive differential pressure sensors by coupled simulated annealing and simplex optimized kernel extreme learning machine," Sensors, vol. 17, no. 4, p. 894, 2017.

[20] Y. T. Fei, Error Theory and Data Processing, China Machine Press, Beijing, China, 1987.

[21] J. Guo and B. X. Zhang, "Numerical investigation of impact erosion in liquid-solid two-phase flow of backfilling pipe," Science \& Technology Review, vol. 33, no. 11, pp. 49-53, 2015.

[22] Z. J. Tang and F. Ren, "Chaotic time series least squares support vector machine prediction algorithm based on iterative error compensation," Acta Physica Sinica, vol. 63, no. 5, pp. 78-87, 2014.

[23] W. Y. Lin and J. Z. Fu, "Thermal error modeling \& compensation of numerical control machine tools based on on-line least squares support vector machine," Computer Integrated Manufacturing Systems, vol. 2, no. 2, pp. 295-299, 2008.

[24] M. M. Adankon and M. Cheriet, "Model selection for the LSSVM. Application to handwriting recognition," Pattern Recognition, vol. 42, no. 12, pp. 3264-3270, 2009.

[25] H. S. Tang, S. T. Xue, R. Chen, and T. Sato, "Online weighted LS-SVM for hysteretic structural system identification," Engineering Structures, vol. 28, no. 12, pp. 1728-1735, 2006. 


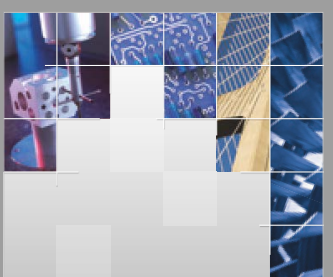

\section{Enfincering}
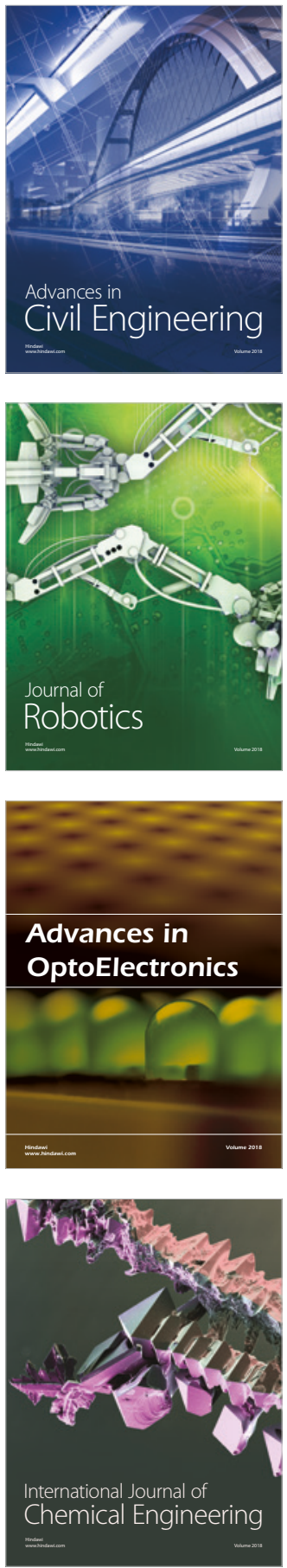

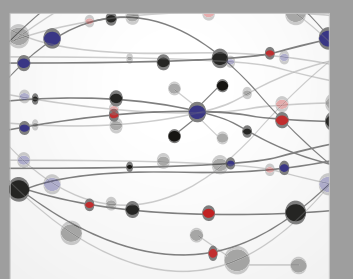

\section{Rotating \\ Machinery}

The Scientific World Journal

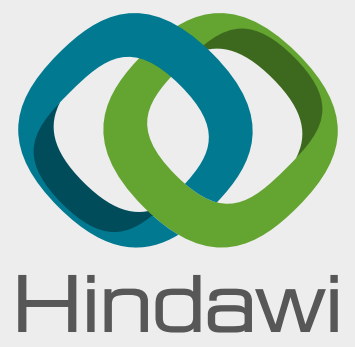

Submit your manuscripts at

www.hindawi.com
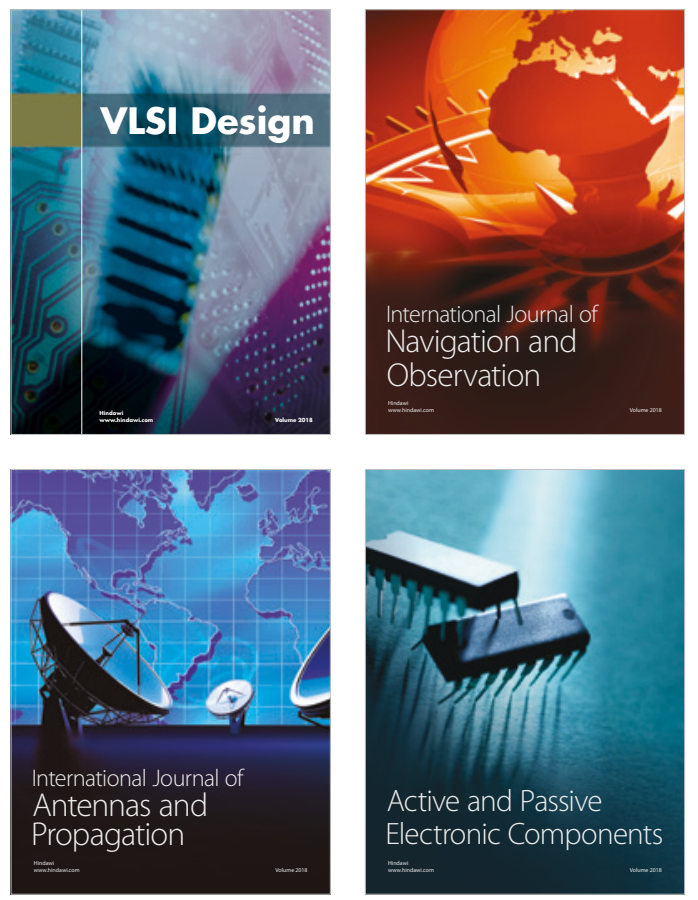
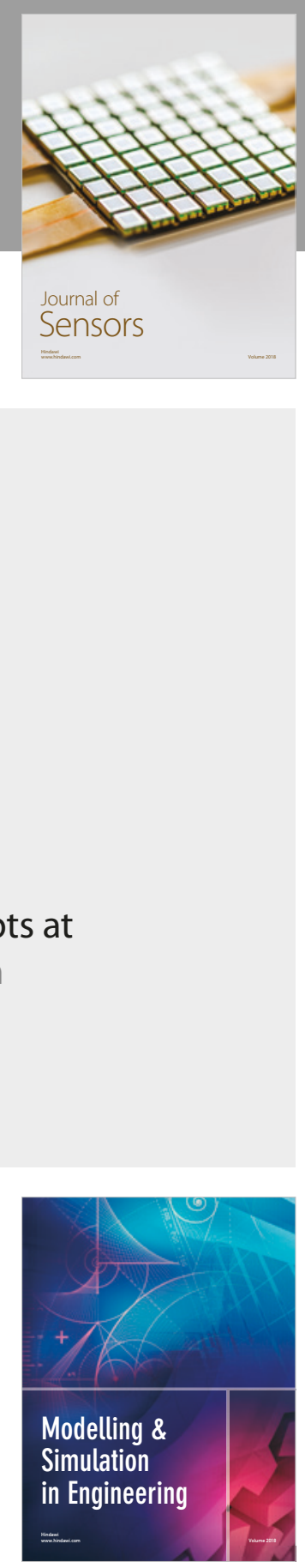

\section{Advances \\ Multimedia}
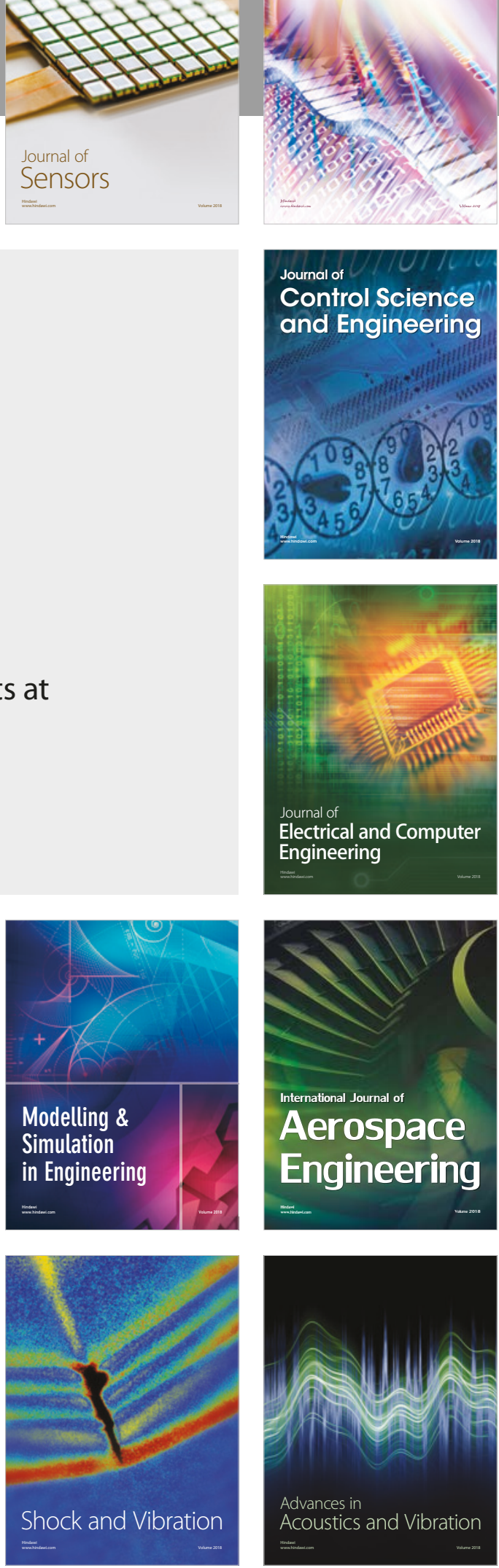